\title{
Évaluation de l'intensité de pluies des espaces à données manquantes
}

\author{
Assessment of rain intensity in locations for which \\ data are missing
}

\author{
I. Juhasz
}

Collaborateur scientifique à l'Institut Hydrographique

Centre de Recherches des Ressources Hydrauliques

(VITUKI) Budapest, Hongrie

\section{Considérations générales}

La connaissance de l'intensité des pluies pour des durées variables $(1 / 2,1,2 \ldots$ heures et $1,2 \ldots 6$ jours $)$ et différentes fréquences, dans un site ou une région est nécessaire pour la recherche hydrologique et pour les applications pratiques.

$\mathrm{Au}$ cours du temps (années, décennies) on a pu accumuler une quantité considérable de données de base. A l'aide d'observations pluviographiques qualitativement et quantitativement convenables et du développement des bases des données d'ordinateur, nous pouvons réaliser des dépouillements complexes soit site par site soit région par région.

Les résultats de ces dépouillements attirent notre attention sur la nature hydroclimatologique des différentes régions et sur les possibilités d'exploitation hydraulique de ces régions.

Notre rapport présente les résultats du dépouillement de données de stations pluviométriques et pluviographiques qui ont permis de développer des formules généralisables; à partir de ces lois, nous pouvons évaluer l'intensité de pluie de zones à données manquantes.

\section{Dépouillement des séries de données}

Les études avaient deux objectifs : d'une part le dépouillement des données des stations pluviométriques et d'autre part le dépouillement des bandes d'enregistrement des stations pluviographiques.

Après avoir dépouillé les données pluviométriques sur de longues périodes (plus de 20 ans) nous avons examiné les répartitions expérimentales des pluies annuelles maximales de durée variable $(1,2 \ldots 6,12$ jours $)$ en appliquant les fonctions de répartition.
D'après les résultats de ces études, les fonctions de répartition expérimentales de pluies annuelles maximales (mm) de durée variable $(1,2 \ldots 6,12$ jours) suivent convenablement la loi log-Normale, ce qui concorde avec les études antérieures $[2,3]$. L'application de lois statistiques différentes, comme la loi de Gumbel et la loi de Pearson (en acceptant des intervalles de confiance de $5 \%$ et en utilisant le test de Sminnov-Kolmogorov) satisfait, dans certaines zones à la loi log-Normale.

Nous avons dépouillé et analysé les bandes d'enregistrement des stations pluviographiques ainsi que les relevés des stations pluviométriques.

Nous avons déterminé les intensités de pluie pour tous les intervalles de 30 minutes depuis le début d'exploitation de la station jusqu'à l'année 1974.

Une partie des enregistrements à été utilisée pour évaluer l'intensité des pluies pour les intervalles de 5 et de 15 minutes. Ainsi nous avons obtenu des séries de données de l'intensité de pluie sur une période de 10 à 20 années d'une dizaine de stations pluviographiques.

Les intensités des pluies de durée variable concernant les courtes et les longues périodes d'observation

$\mathrm{Au}$ cours du dépouillement des enregistrements pluviographiques, nous avons déterminé pour toutes les stations, les valeurs de différentes fréquences et les moyennes des intensités annuelles maxima des pluies de durée variable $(1 / 2,1,2, \ldots 12$ heures et $1,2, \ldots 6$, 12 jours) pour la période d'analyse.

Les valeurs, qui donnent l'intensité en fonction du temps (Fig. 1) s'alignent sur une droite. Ceci signifie que les intensités des durées variables $(1 / 2,1 \ldots$ heures 


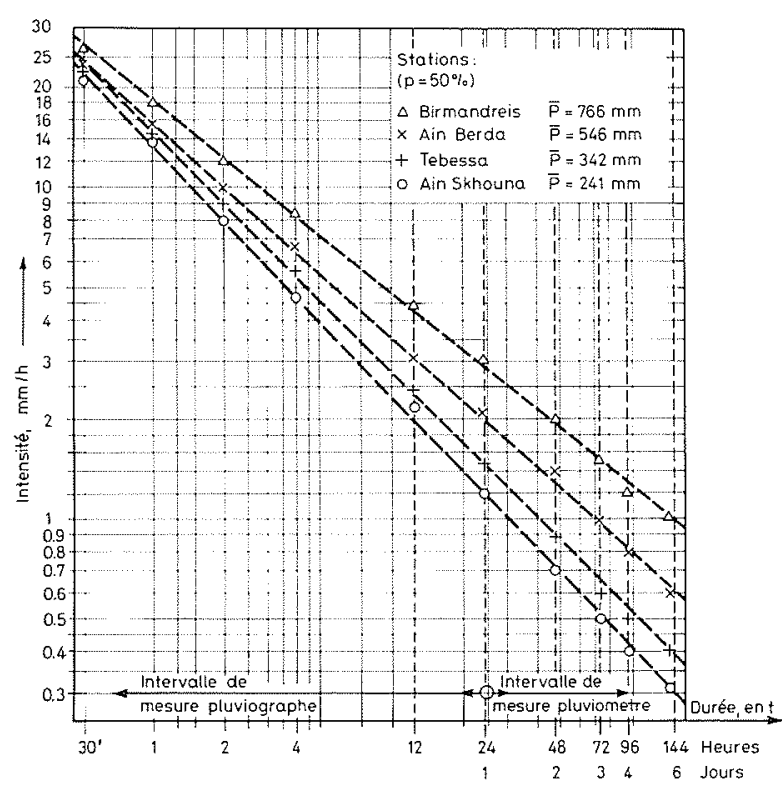

Figure 1. - Lois statistiques des intensités de la pluie des postes pluviométriques en Algérie (Période de retour 2 ans).

jusqu'à 12 jours) d'une station suivent une loi représentée par une seule fonction. Oil trouvera des résultats similaires dans l'étude de Guyon [4].

Ces résultats concernant les stations pluviographiques sont extrapolables aux stations pluviométriques où l'on dispose des relevés journaliers pour une période d'observation convenablement longue. C'est dire qu'à partir de cette loi, l'extrapolation de la fonction de l'intensité des pluies de longue durée (de 1 à plusieurs jours) permet de déterminer l'intensité des pluies de courte durée, inférieure à un jour, pour le poste pluviométrique.

Les paramètres de la fonction de l'intensité des pluies de durée variable des différentes stations pluvio-

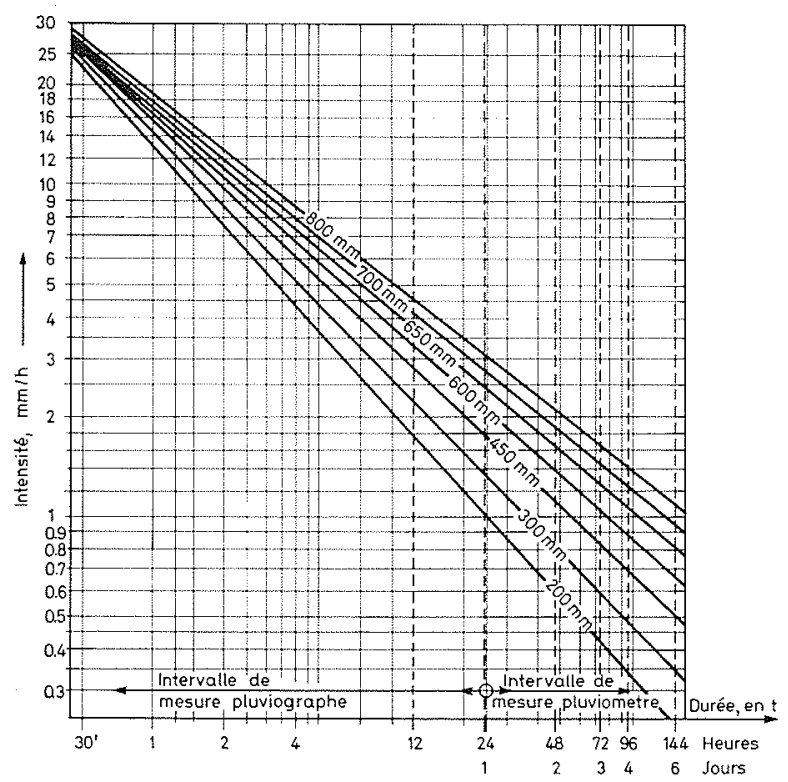

Figure 2. - Loi statistiques des intensités de la pluie en Algérie en fonction de la pluviométrie interannuelle (Période de retour 2 ans). métriques sont fortement correlés à la pluviométrie moyenne interannuelle.

Nous avons constaté que la pente de la fonction de l'intensité des pluies de durée variable est inversement proportionnelle à la valeur de la pluie moyenne annuelle.

Plus la pente de la fonction de l'intensité des pluies est forte plus la pluie moyenne annuelle est faible et vice-versa. (Fig. 2).

D'après nos expériences, les fonctions de différentes pluviométries moyennes convergent vers une même valeur d'intensité, mais l'analyse physique de cette convergence fera l'objet d'une étude ultérieure. A partir de ces résultats, nous pouvons évaluer l'intensité de la pluie pour une site donné où nous ne disposons que de la pluie moyenne annuelle représentée par exemple sur une carte de la pluviométrie interannuelle.

\section{Evaluation de l'intensité de pluie des zones à données manquantes}

Nous avons appliqué les résultats de nos études, aux calculs hydrauliques de différentes régions climatiques en Algérie [5].

Notre application de l'évaluation de l'intensité des pluies de durée variable concerne deux sortes de cas : - utilisation des relevés de postes pluviométriques similaires et voisins pour déterminer la loi de l'intensité de pluie de longue durée et extrapolation pour l'intensité de pluie de courte durée (quelques heure) à l'aide de la figure 1 ;

- utilisation d'une carte de pluviométrie interannuelle pour évaluer la pluie moyenne du site, et utilisation des résultats des lois statistiques de la zone (Fig. 2) pour estimer l'intensité des pluies de durée variable.

Pour les études spéciales des intensités des pluies de durée variable on peut élaborer les cartes:

- des valeurs des pentes de la fonction de l'intensité de pluie, et

- des valeurs de pluie de $24 \mathrm{~h}$ de différentes fréquences.

A partir de ces cartes, les intensités sont évaluables.

\section{Remerciements}

Les résultats pratiques de ce rapport sont le résultat d'une coopération de longue durée de VITUKI (Budapest) et de la DEMRH (Alger).

Je tiens à remercier Monsieur K. Achi, Directeur de la DEMRH, et Monsieur A. Demmak, chef du Service Hydrologie de la DEMRH pour ses encouragements lors de l'élaboration de cet article : ainsi que Monsieur K. Futaki et Monsieur $Z$. Vargay, collaborateurs de VITUKI, pour leur coopération de l'élaboration des programmes de calcul par ordinateur et mes chers collègues algériens et hongrois pour leur aide. 


\section{Bibliographie}

[1] MEDINGER G. - Hauteurs de pluie - Moyenne de 40 ans S.E.S., Alger, 1955.

[2] CHAUMONT M. et PAQUIN C. - Notice explicative de la carte pluviométrique de l'Algérie au 1/500.000. C.C.R.M.A., Alger, 1971.
[3] MASSON J.M. - Etude d'homogeneisation (Rapport d'activité. D.E.M.R.H., Alger, 1974.

[4] GUYON G. - Les calculs d'un projet de drainage, Bull. Technique de Génie Rural. C.T.G.R.E.F., n 123, 1978, Paris-Antony.

[5] JUHASZ I. - Etude pluviométrique régionale, Wilaya de Constantine, n 80, DEMRH, Alger, 1978.

\section{Discussion}

Le Président. - Monsieur DERI, je vous remercie beaucoup pour cette communication remarquable, et fort utile pour ceux qui ont besoin de faire des calculs hydrologiques.

Avant de passer à la discussion, je voudrais faire deux remarques.

La première est qu'il est fort intéressant d'avoir, au cours d'une même manifestation, deux communications qui s'attaquent finalement au même problème, l'une sur la Tunisie, ce matin, l'autre sur l'Algérie, un pays voisin, ce, avec des méthodes qui sont tout de même très différentes. Il y a là des possibilités de comparaison intéressantes quí peuvent susciter quelques commentaires.

Ma deuxième remarque est à l'adresse de VITUKI. Dans les études de l'ORSTOM sur le même sujet, aussi bien en Afrique de l'Ouest que pour la Martinique et la Guadeloupe, nous avons adopté la même méthode que vous; elle paraît peut-être moins scientifique que l'analyse extrêmement détaillée faite par l'institut de Toulouse, mais finalement, répond bien aux problèmes qui se posent aux ingénieurs en matière de répartition des in tensités.

Je vais maintenant demander si quelqu'un souhaite prendre la parole.

M. THIRRIOT. - Après la présentation de M. DERI, je note avec plaisir la proximité géographique et scientifique des préoccupations de nos collégues hongrois et celles que nous avons à l'ENIT. Et il me semble qu'il y aura pour nous un grand profit dans la comparaison des résultats.

M. GIRAUID. -. I.e comportement asymptotique des lois normales étant fort différent de la loi de Gumbel, je voudrais demander si le choix de la loi log-normale est un a priori que l'expérience a confirmé ou si c'est le comportement asymptotique des échantillons qui a conduit à choisir une loi lognormale.

M. DERI. - On a fait plusieurs expérimentations pour le meilleur choix. Si l'on prend en considération le critère statistique de décision du choix, dans ce cas, pratiquement, il n'y a pas de différence entre les deux fonctions de répartition, si l'on considère la bande de l'intervalle de confiance, c'est-à-dire que, pour toutes les utilisations, les deux fonctions de répartition permettent un certain arbitrage du choix.

C'est pourquoi je suis d'accord, par exemple, avec l'application de la fonction de répartition de $M$. THIRRIOT, parce que l'application de cette fonction aboutit pratiquement au même résultat.

Le Président. - Si personne ne veut intervenir, je voudrais ajouter quelques mots à la suite de ce que viennent de dire à la fois M. GIRAUD et M. DERI.

Dans l'étude de M. THIRRIOT, plusieurs méthodes sont mises en cuvre, dont une transformation d'échantillons par anamorphose. Cela résoud apparemment le problème du choix du modèle de distribution, puisque, par anamorphose, on crée un nouvel échantillon obéissant à une loi choisie a priori. En réalité, le problème est simplement déplacé et la méthode présente tout de même un inconvénient : l'interprétation des résultats est plus délicate qu'avec l'échantillon naturel.

La pratique est bonne, mais il faut faire attention : toutes les statistiques qu'on peut tirer des échantillons transformés sont en fait plus difficiles à interpréter, puisque ce ne sont plus les valeurs naturelles sur lesquelles on travaille.

Pour répondre à M. GIRAUD, il est indiqué, dans la communication qui vient d'être présentée, que le test d'adéquation choisi, le test de Smirnov-Kolmogorov, est bon surtout dans les parties centrales des distributions : il l'est peut-être un peu moins pour les valeurs extrêmes, mais tout le monde sait que, quand il s'agit de définit des ajustements, d'une part, et des choix d'adéquation d'autre part, pour les branches extrêmes d'une distribution, tout le monde est logé à la même enseigne ; il faut prendre les choses un peu comme elles sont et ne pas trop se faire d'illusions sur les résultats.

M. DERI. - Je voudrais vous remercier pour les questions et pour les paroles d'appréciation de M. ROCHE.

Permettez-moi d'ajouter une seule phrase : il y a une certaine expérience en ce qui concerne l'analyse de sensibilité de l'estimation des paramètres pluviométriques en fonction de la taille de l'échantillon, et je crois que ce sera utile pour les recherches futures. Si on a de la chance en ce qui concerne la validité des échantillons, de temps en temps, il suffit d'une série d'observations de dix ans pour bien estimer les paramètres de la fonction de répartition.

Le Président. - Je remercie beaucoup M. DERI, et nous allons passer à la communication suivante, concernant les variations pluviométriques durant la dernière période séculaire sur la façade occidentale de l'Europe. 


\section{Abstract \\ Assessment of rain intensity in locations for which data are missing}

The results obtained by processing the data of conventional and recording rain gages are presented. These were used for deriving a relationship of general validity by which rain intensities can be estimated for certain regions with incomplete data.

The data of rain gages with long (round 20 years) records available were examined to approximate the empirical distributions of annual greatest rainfalls of different $(1,2, \ldots 6,12$ days) durations by different distribution functions.

Along with the observation data, the charts of recording rain gages were also processed and analysed, determining as accurately as the chart would permit the rain intensities by $30^{\prime}$ time intervals for the period on record up to 1974. (During the last years the $30^{\prime}$ intervals could be resolved into 5 and 15 minutes) In the way, 10-20 years intensity time series were produced for round 10 stations.
From the charts of the individual stations, we have determined the rain intensities of different probability during the annual maxima of $0.5,1,2 \ldots$ hours and $1,2, \ldots 6,12$ days duration. In an intensity vs. time diagram these values plotted to a straight line (Fig. 1), implying that the rain intensities of short (few hours) and longer (few days) duration are described by a single function at a particular station.

This result can be extended to rain gaging stations (having no recording gage), where a sufficiently long observation record is available. By extrapolating the function it is thus possible to estimate the intensity of rains shorter than one day for a particular station.

The slope of the rain intensity functions was found to be related to the normal average annual rainfall at the station. The functions at stations with little annual rainfall were very steep relative to the flat functions of the stations with abundant rainfall (Fig. 2). 